\title{
Gut Lymphangiopathy: Adding Fuel to the Fire in Chronic Liver Disease
}

\author{
Luis Tresierra1, Maria-Angeles Aller ${ }^{2}$, Isabel Prieto ${ }^{3}$, Luis Santamaria ${ }^{4}$, Jaime Arias ${ }^{2}$ \\ ${ }^{1}$ Department of Surgery, Sant Joan Despi Moises Broggi Hospital, Barcelona, Spain \\ ${ }^{2}$ Department of Surgery, School of Medicine, Complutense University of Madrid, Madrid, Spain \\ ${ }^{3}$ Department of General and Digestive Surgery, La Paz Hospital, Autonoma University of Madrid, Madrid, Spain \\ ${ }^{4}$ Department of Anatomy, Histology and Neuroscience, School of Medicine, Autonoma University of Madrid, Madrid, Spain \\ Email: 1tresierra@gmail.com, `maaller@med.ucm.es, jariasp@med.ucm.es, iprieto@intermic.com, luis.santama ria@uam.es
}

How to cite this paper: Tresierra, L., Aller, M.-A., Prieto, I., Santamaria, L. and Arias, J. (2019) Gut Lymphangiopathy: Adding Fuel to the Fire in Chronic Liver Disease. Advances in Bioscience and Biotechnology, 10, 305-319.

https://doi.org/10.4236/abb.2019.1010024

Received: July 2, 2019

Accepted: October 12, 2019

Published: October 15, 2019

Copyright $\odot 2019$ by author(s) and Scientific Research Publishing Inc. This work is licensed under the Creative Commons Attribution International License (CC BY 4.0).

http://creativecommons.org/licenses/by/4.0/

\section{(c) (i) Open Access}

\begin{abstract}
The splanchnic inflammation inchronic liver disease increases intestinal angiogenesis. In the current study our aim was demonstrating that the small bowel lymphangiogenesis is associated with angiogenesis in chronic cholestasis in the rat. A stereological study of the lymphatic microcirculation in the small intestine was performed in cholestatic rats. Portal enteropathy in cholestasis increases lymphatic microvessels in the mucosa and submucosa layers. Thus, the lymphatic microvessel volume fraction was superior $(\mathrm{p}<0.001)$ in the mucosa $(0.16 \pm 0.01)$ and submucosa $(0.16 \pm 0.01)$, in regard to the muscle layer $0.015 \pm 0.01$. The lymphatic microvessel length density was higher in the mucosa $\left(76.89 \pm 2.86 \mathrm{~mm}^{-2} ; \mathrm{p}<0.001\right)$ and submucosa $(14.87 \pm 2.86$ $\left.\mathrm{mm}^{-2} ; \mathrm{p}<0.01\right)$, in relationship to the muscle layer $\left(5.04 \pm 2.92 \mathrm{~mm}^{-2}\right)$. These alterations predominate in the duodenum (volume fraction: $0.10 \pm 0.01$ and length density: $33.55 \pm 5.98 \mathrm{~mm}^{-2}$ ) and ileum (volume fraction: $0.16 \pm 0.01$ and length density: $38.62 \pm 6.07 \mathrm{~mm}^{-2}$ ). This study demonstrates the predominance of an increased lymphangiogenic response in both end sides of the small bowel associated with chronic liver disease. Since the porto-systemic venous collateral circulation in the chronic liver insufficiency is developed in the ends of the gastrointestinal tract, the excessive duodeno-ileal lymphangiogenesis could suggest the development of amesenteric-systemic lymphatic bypass in the chronic portal hypertension.
\end{abstract}

\section{Keywords}

Chronic Liver Disease, Lymphangiogenesis, Microsurgery, Collateral Lymphatic Circulation

\section{Introduction}

The basic structural impairment found in the gastrointestinal tract in portal 
hypertension is vascular and consists of a greater number and larger blood vessels [1] [2]. Consequently, the name proposed for this kind of enteropathy is "hypertensive portal intestinal vasculopathy" [3].

In addition, sustained portal hyperpressure increases mesenteric lymph flow and produces impairment of lymphatic drainage, which is considered an important etiopathogenic mechanism of ascites [4] [5]. In turn, chronic functional failure of the gastrointestinal lymphatic vascular system in portal hypertension induces a compensatory lymphangiogenic response [5] [6]. At present, the characteristics of intestinal lymphatic vascular alterations typical of portal hypertension are unknown, especially when associated with chronic progressive liver failure [6]. Consequently, one of the objectives of this study is to demonstrate the distribution of intestinal lymphatic vascular changes in an experimental model of chronic portal hypertension secondary to biliary fibrosis.

Since splanchnic alterations associated with chronic liver failure are considered inflammatory in nature [7] [8], it could be proposed that, similar to the mesenteric venous vasculopathy, a hypertensive intestinal lymphangiopathy is developed associated with biliary fibrosis in rats, which would also have a similar pathogenesis.

\section{Material and Methods}

\subsection{Animal Management}

Male Wistar rats, with an average weight between 218 and 334 g, corresponding to an age from 8 to 10 weeks from the Animal Facility of the experimental surgery unit of La Paz University Hospital were used.

The animals were fed a standard laboratory rodent diet (rat/mouse A 04 maintenance diet; Panlab. Barcelona. Spain) and water ad libitum. They were housed in a light/dark-controlled room (light cycles: 7:30 a.m. - 7:30 p.m.) with an average temperature $\left(22^{\circ} \mathrm{C} \pm 2^{\circ} \mathrm{C}\right)$ and humidity $(65 \%-70 \%)$, in groups of three to four animals.

The experimental procedures employed in this study were based on the principles and practices of the 1986 European Guide for the Care and Use of Laboratory Animals, in accordance with the Ethical Guidelines from European Community Council Directive (86/609/EEC) and published in Spanish Royal Decree 1201/2005. All procedures were approved by the La Paz Hospital Animal Welfare Committee.

\subsection{Experimental Design}

Forty-one male rats were randomized and divided into two groups: Sham-operated (SO; $\mathrm{n}=11)$, microsurgical cholestasis $(\mathrm{MC} ; \mathrm{n}=30$ ). In the SO-group, all the animals survived and in the MC-group, five rats died by gastrointestinal hemorrhage related to hepatic failure in the seventh week of postoperative evolution. Therefore, thirty-six of the rats from the beginning of the study survived until the time of sacrifice: Sham-operated $(\mathrm{SO} ; \mathrm{n}=11)$ and microsurgical cholestasis (MC; $\mathrm{n}=25)$. 


\subsection{Surgical Procedure}

The rats were anesthetized with $1.5 \%$ - $2 \%$ Isofluorane (inhalatory vaporizer, TEC 4 (Ohemeda). Surgery was performed under aseptic, but not sterile, conditions.

In the SO rats, the bile duct and its lobular branches were dissected without resection. In the MC rats, an extrahepatic biliary tract resection was performed using a binocular operatory microscope (OPMI 1-FR; Zeiss, Madrid, Spain), as previously described [9] [10]. Briefly, the common bile duct was ligated (silk 4/0) and sectioned and the lobular biliary branches are ligated and then sectioned.

The abdomen was closed in two layers with an absorbable suture (3/0 polyglycolic acid) and silk (3/0). Buprenorphine ( $0.05 \mathrm{mg} / \mathrm{kg} / 8$ h s.c.) was administered for analgesia in the early postoperative period. Also a broad spectrum antibiotic (Ceftriaxona; $50 \mathrm{mg} / \mathrm{kg}$, twice a week) and vitamin K1 (Phytomenadione; $8 \mathrm{mg} / \mathrm{kg}$, once a week) were administered.

All the rats were sacrificed at $8^{\text {th }}$ week of intervention. The following aspects were studied:

\subsection{Ascites Volume Determination}

The extraction of ascitic fluid was performed in all the cholestatic rats by aspiration using a $10 \mathrm{ml}$ syringe.

\subsection{Portosystemic Collateral Circulation}

First, a middle abdominal incision was performed. Next, the areas where the collateral venous circulation develops, that is, splenorenal, gastroesophageal, and colorectal, were studied for the presence of collateral veins [7].

\subsection{Serum Biochemical Tests}

In the blood obtained by puncture of the infrahepatic inferior vena cava, bilirubin, alkaline phosphatase, Aspartate-aminotransferase (AST), Alanine-aminotransferase (ALT) and Albumin serum concentrations were measured with commercially available kits (Sigma), using a Hitachi U-2000 spectrophotometer (Hitachi Nissei Sangyo Co. Ltd., Japan).

\subsection{Immunolabelling of the Intestinal Lymphatic Vessels with LYVE-1}

Lymphatic endothelial cells share many markers in common with vascular endothelial cells [11]. Cell surface markers specific of lymphatic endothelium have been developed. Lymphatic endothelial cells have been isolated by positive selection using antibodies to podoplanin, vascular endothelial growth factor receptor (VEGFR)-3 or lymphatic vessel hyaluronan receptor (LYVE)-1, and by a negative selection with antibodies to CD34 [12]. Furthermore, the transcriptional factor prospero-related homeodomain transcription factor (Prox)-1 is essential for establishing lymphatic endothelial cell identity; its expression is controlled by 
Sox 18 transcription factor [13] [14]. Lymphatic endothelial cells express specific molecules such as Prox-1, VEGFR-3, the membrane glycoprotein podoplanin and LYVE-1 [15]; the latter was used as a cellular lymphatic marker in the present stereological study (Figure 1).

Samples of small intestine (duodenum, jejunum, and ileum) were obtained from all the groups, fixed in paraformaldehyde (10\%) for 1 week, dehydrated in ethanol, and embedded in paraffin. Paraffin sections (2 - $4 \mu \mathrm{m}$ thick) were cut and attached to glass slides. Some deparaffinized sections were stained with hematoxylin-eosin (HE) to show morphological findings. The other deparaffinized sections were rehydrated in phosphate-buffered saline (PBS) and immunostained [16]. Briefly, all serial sections for the immunoperoxidase staining were first blocked with $1 \%$ hydrogen peroxide in PBS. Next, they were incubated in a blocking solution, a PBS with 5\% normal goat serum (NGS) (Vector Laboratories; Burlingame, CA, USA) for 1 hour at room temperature. Then they were immunostained with rabbit polyclonal anti-LYVE-1, dilution 1:500 (Abcam; Cambridge, UK), in PBS with 5\% NGS at $4^{\circ} \mathrm{C}$ overnight. The immunostained sections were finally incubated in biotin-conjugated goat anti-rabbit IgG (Vector Lab.) at RT for 1 hour. The immunoreaction products were later visualized with Vectastain $^{\circledR}$ avidin-biotin complex ( $\mathrm{ABC}$ ) reagent (Vector Lab.) and a metal-enhanced diaminobenzidine (DAB) substrate kit (Pierce; Rockford, IL, USA) [17]. After immunoreactions, the sections were counterstained with Harris hematoxylin. All the slides were dehydrated in ethanol and mounted in a synthetic resin (Depex, Serva, Heidelberg, Germany).

\subsection{Quantitative Methods for Studying the Intestinal Lymphatic Vessels}

The following parameters were assessed in all groups of animals:

1) Volume fraction occupied by lymphatic vessels ( $V_{V}$ vasc).

$$
V_{v} \text { vasc }=\frac{\sum \text { points on vessels }}{\sum \text { points on reference space }}
$$

2) Length density of lymphatic vessels $\left(L_{v}\right)$. Dissector frame was used to sample immunostained vascular profiles eligible to be counted by the Sterio rule [18]. The length density is calculated using the formula:

$$
L_{v}=\frac{\left(2 \times \sum Q-\right)}{\sum A}
$$

where Q- = number of eligible immunopositive vascular profiles and

$\sum A=$ stromal area sampled, i.e., area of dissector frame, multiplied by the number of selected frames.

$L_{\mathrm{v}}=$ was expressed in $\mathrm{mm}^{-2}$ ( $\mathrm{mm}$ length of vessel $/ \mathrm{mm}^{3}$ reference volume).

3) Average vascular diameter (AVD) calculated according to the formula and expressed in $\mu \mathrm{m}$ [18]:

$$
\text { avd }=2 \times 103 \times \sqrt{V_{v} \operatorname{vasc} / V_{v} \operatorname{stroma} \cdot \pi \cdot L_{v}}
$$



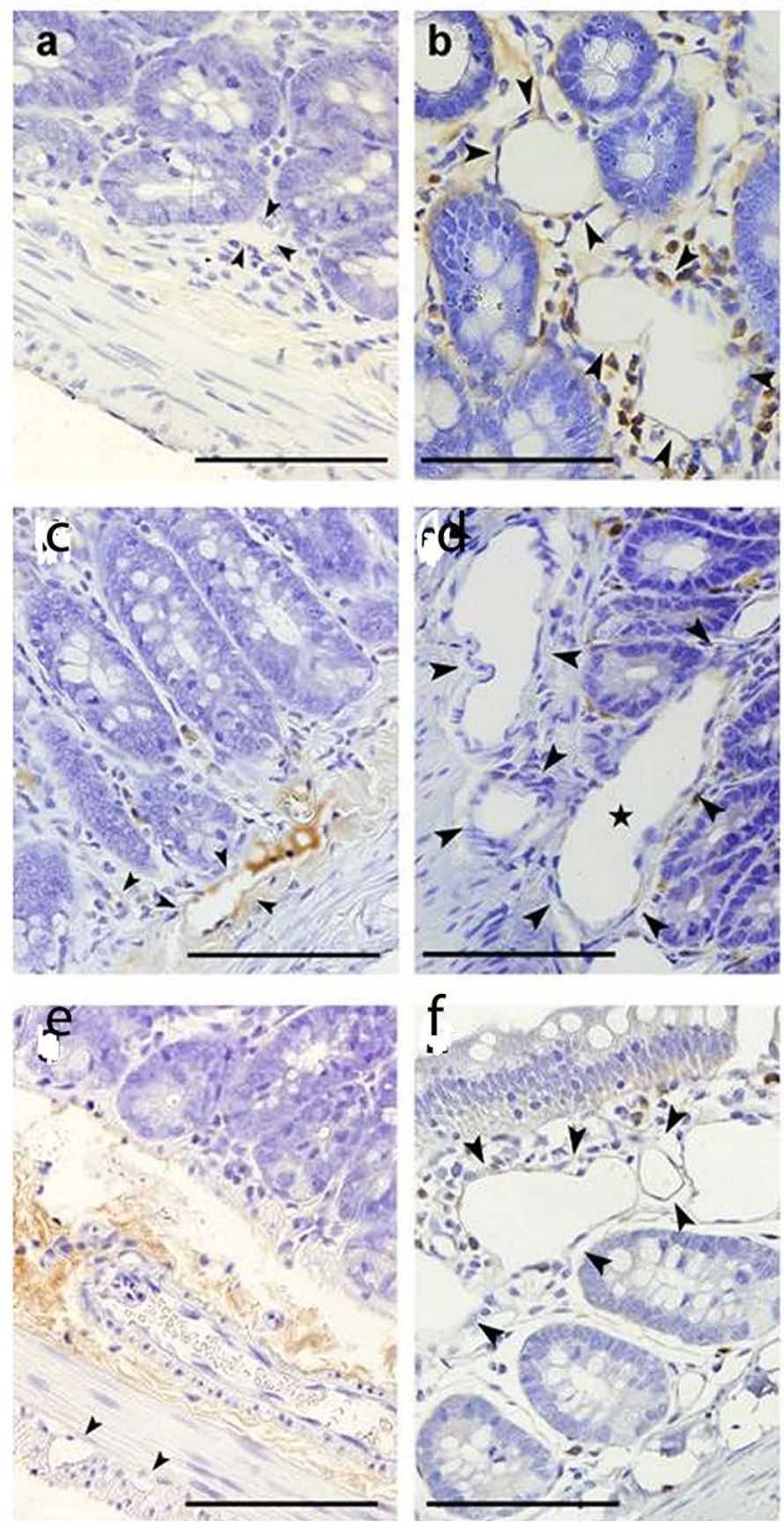

Figure 1. Small intestine of sham operated (SO) and microsurgical cholestatic-rats (MC) immunostained with LYVE1. (a) Image of a duodenum SO-rat: a small lymphatic lumen is observed in the lamina propria (arrowheads); (b) Duodenal lamina propria from a rat with MC: abundant and enlarged lymphatics are observed (arrowheads); (c) Image of a jejunum SO-rat: a lymphatic lumen is observed in submucosa (arrowheads); (d) lamina propria of jejunum from a rat with MC: abundant and enlarged lymphatics are observed in lamina propria (star) and submucosa (arrowheads); (e) Ileal image from a SO-rat: two small lymphatic lumina are visualized (arrowheads); (f) Ileal mucosa from an animal with MC showing a relevant enlargement of lymphatic microvessels (arrowheads). The size of the calibration bars throughout all images was $100 \mu \mathrm{m}$. 


\subsection{Statistical Analysis}

The data was analyzed using a statistical package for social sciences (SPSS, version 21, Chicago, Inc.). Mean values \pm SEM were obtained from stereological parameters. 2-factor ANOVA and a post-hoc Duncan test were performed for statistical comparison. A statistical significance was assumed if $\mathrm{p}<0.05$.

\section{Results}

\subsection{Body and Organ Weights}

Liver and spleen weight in the animals with MC increased ( $\mathrm{p}<0.001)$ with respect to the SO animals. On the contrary, the testicular weight of rats with $\mathrm{MC}$ decreased $(\mathrm{p}<0.05)$ with respect to the SO rats (Table 1$)$.

\subsection{Portosystemic Collateral Circulation}

All the cholestatic rats developed portosystemic collateral circulation, whether pararectal, paraesophageal, or splenorenal.

\subsection{Ascites Volume}

The mean volume of ascitic fluid in the group with MC was $10.5 \pm 8.6 \mathrm{ml}$.

\subsection{Liver Function}

The animals with MC had an increase $(\mathrm{p}<0.001)$ of total bilirubin, alkaline phosphatase, AST and ALT serum levels with respect to the SO-rats (Table 2). Albumin serum level was lower $(\mathrm{p}<0.001)$ in MC-rats compared to SO-rats (Table 2).

\subsection{Stereological Study of the Lymphatic Microcirculation in Microsurgical Obstructive Cholestasis}

The rats with microsurgical cholestasis displayed an increase in volume fraction and length density of the lymphatic vessels located in mucosa $\left(\mathrm{V}_{\mathrm{v}}\right.$ vasc: $0.16 \pm$ $\left.0.01 ; \mathrm{L}_{\mathrm{v}}: 76.89 \pm 2.86 \mathrm{~mm}^{-2}\right)$ and submucosa $\left(\mathrm{V}_{\mathrm{v}}\right.$ vasc: $0.16 \pm 0.01 ; \mathrm{L}_{\mathrm{v}}: 14.87 \pm$ $2.86 \mathrm{~mm}^{-2}$ ) in relation to the SO-rats (Figure 2(a), Figure 2(b) and Figure 3(a) and Figure 3(b)). Thus, these parameters increased in the duodenum $\left(\mathrm{V}_{\mathrm{v}}\right.$ vasc: $0.10 \pm 0.01$ vs. $\mathrm{V}_{\mathrm{v}}$ vasc: $0.04 \pm 0.01 ; \mathrm{p}<0.001 ; \mathrm{L}_{\mathrm{v}}: 33.55 \pm 5.98 \mathrm{~mm}^{-2}$ vs. $\mathrm{L}_{\mathrm{v}}: 8.68 \pm$

Table 1. Liver weight (LW), Spleen weight (SW) and Testes weight (TW) in Sham-operated rats (SO) and in rats with microsurgical cholestasis (MC).

\begin{tabular}{cccc}
\hline & LW $(\mathrm{g})$ & SW $(\mathrm{g})$ & TW $(\mathrm{g})$ \\
\hline SO & & & \\
$(\mathrm{n}=11)$ & $11.77 \pm 1.22$ & $0.82 \pm 0.14$ & $3.26 \pm 0.18$ \\
\hline MC & & \\
$(\mathrm{n}=25)$ & $19.81 \pm 1.95^{* * *}$ & $1.69 \pm 0.28^{* *}$ & $2.01 \pm 0.09^{*}$
\end{tabular}

Mean $\pm \mathrm{SE} ;{ }^{*} \mathrm{p}<0.05 ;{ }^{* *} \mathrm{p}<0.01 ;{ }^{* * *} \mathrm{p}<0.001$ : statistically significant value with respect to SO-rats. 
Table 2. Serum concentrations of aspartate aminotransferase (AST; U/I), alanine aminotransferase (ALT; U/I), and Albumin (mg/dl) in Sham operated rats (SO) and in rats with microsurgical cholestasis (MC).

\begin{tabular}{ccccc}
\hline TB $(\mathrm{mg} / \mathrm{dl})$ & AP $(\mathrm{U} / \mathrm{L})$ & AST $(\mathrm{U} / \mathrm{L})$ & ALT $(\mathrm{U} / \mathrm{I})$ & Albumin \\
\hline SO $0.09 \pm 0.04$ & & & & \\
$(\mathbf{n}=11)$ & $112.34 \pm 34.12$ & $105.75 \pm 38.35$ & $45.75 \pm 12.52$ & $3.09 \pm 0.33$ \\
\hline MC $8.17 \pm 1.76^{* * *}$ & & & & \\
$(\mathbf{n}=25)$ & $284.88 \pm 69.30^{* * *}$ & $484.96 \pm 36.76^{* * *}$ & $100.75 \pm 45.08^{* * *}$ & $1.09 \pm 0.23^{* * *}$ \\
\hline
\end{tabular}

Mean $\pm \mathrm{SE} ;{ }^{* * *} \mathrm{p}<0.001$ : statistically significant value with respect to SO-rats.

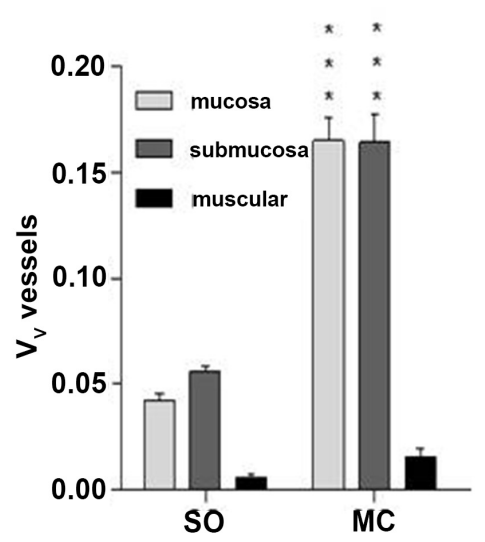

(a)

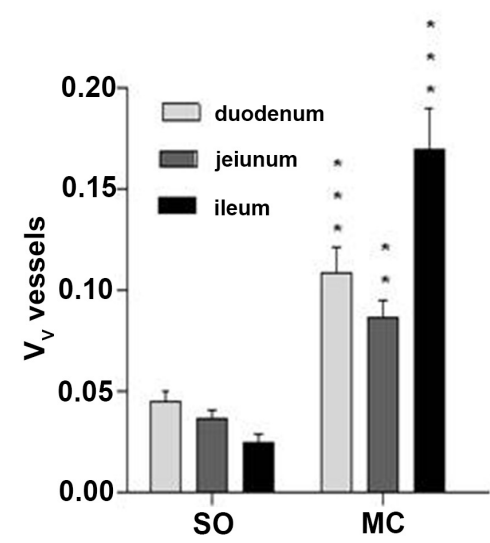

(b)

Figure 2. Stereologic quantification of the lymphatic vessel volume fraction (Vv) according small bowel layers (a) (mucosa, submucosa and muscular) and regions (b) (duodenum, jejunum and ileum). SO (Sham-operated rats); MC (rats microsurgical cholestasis); the results are expressed as mean $\pm \mathrm{SD} .{ }^{* *} \mathrm{p}<0.01 ;{ }^{* *} \mathrm{p}<0.001$ : statistically significant with respect to SO rats.

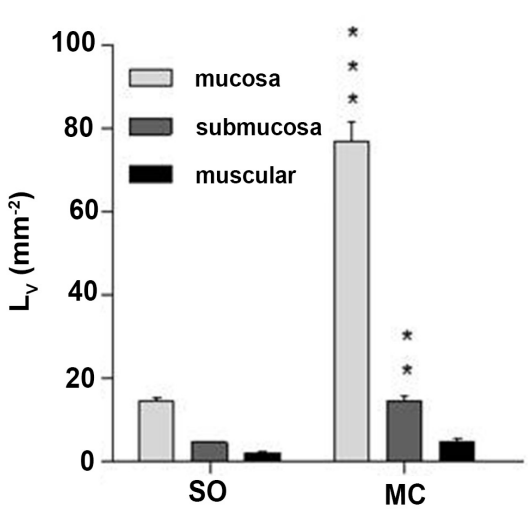

(a)

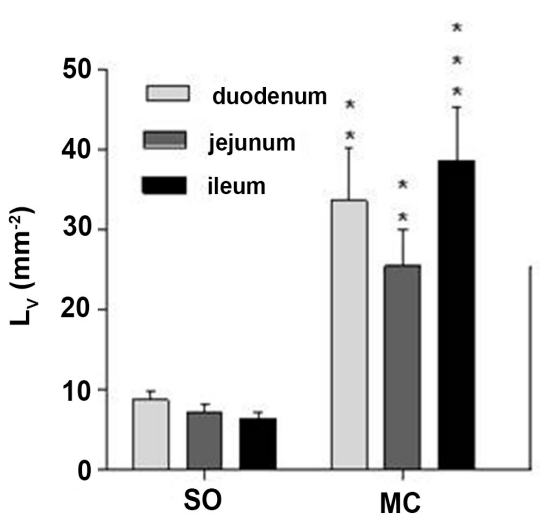

(b)

Figure 3. Stereological quantification of the lymphatic vessels length (Lv) density according small bowel layers (a) (mucosa, submucosa and muscular) and regions (b) (duodenum, jejunum and ileum). SO (Sham-operated rats); MC (rats with microsurgical cholestasis); the results are expressed as mean $\pm \mathrm{SD} .{ }^{* *} \mathrm{p}<0.01 ;{ }^{* *} \mathrm{p}<0.001$ : statistically significant value in regards to SO rats; $\mathrm{p}<0.05 ; \mathrm{P}<0.001$ : statistically significant value in regards to MC rats. 
$\left.5.98 \mathrm{~mm}^{-2} ; \mathrm{p}<0.001\right)$ jejunum ( $\mathrm{V}_{\mathrm{v}}$ vasc: $0.08 \pm 0.01$ vs. $\mathrm{V}_{\mathrm{v}}$ vasc: $0.03 \pm 0.01 ; \mathrm{p}<$ $0.01 ; \mathrm{L}_{\mathrm{v}}: 25.42 \pm 5.98 \mathrm{~mm}^{-2}$ vs. $\left.\mathrm{L}_{\mathrm{v}}: 7.02 \pm 5.98 \mathrm{~mm}^{-2} ; \mathrm{p}<0.01\right)$ and ileum $\left(\mathrm{V}_{\mathrm{v}}\right.$ vasc: $0.16 \pm 0.01$ vs. $\mathrm{V}_{\mathrm{v}}$ vasc: $0.02 \pm 0.01 ; \mathrm{p}<0.001 ; \mathrm{L}_{\mathrm{v}}: 38.63 \pm 6.07 \mathrm{~mm}^{-2}$ vs. $\mathrm{L}_{\mathrm{v}}$ : $6.26 \pm 6.07 \mathrm{~mm}^{-2} ; \mathrm{p}<0.001$ ), of the cholestatic rats in relationship to SO-rats, although these increases are more significant in duodenum and ileum (Figure 1(b) and Figure 1(e); Figure 2(b) and Figure 3(b)).

Finally, the average vascular diameter (AVD) was similar either in all the layers as in each anatomical area (duodenum, jejunum and ileum) of the small bowel in the SO-rats and in the cholestatic rats.

\section{Discussion}

This experimental study used stereological methods in combination with a cellular surface marker for lymphatic endothelial cells to estimate such parameters as volume fraction, length density, and average vascular diameter of lymphatic vessels [19] [20] in the small intestine of microsurgical cholestatic rats.

Lymphatic endothelial cells share many markers [11], which have been isolated by positive selection using antibodies to podoplanin, vascular endothelial growth factor receptor (VEGFR)-3 or lymphatic vessel hyaluronan receptor (LYVE)-1, and by a negative selection with antibodies to CD34 [12]. The Prox-1 is essential for establishing lymphatic endothelial cell identity; its expression is controlled by Sox 18 transcription factor [13] [14]. Lymphatic endothelial cells express specific molecules such as Prox-1, VEGFR-3, the membrane glycoprotein podoplanin and LYVE-1 [15].

The increased volume fraction and length density of lymphatic vessels in the small intestine mucosa and submucosa in this experimental model of microsurgical biliary fibrosis could be attributed to increased interstitial lymph production which, in turn, produces increased lymph flow and pressure in the lymphatic microvessels. Moreover, intestinal lymphatic vessel dysfunction could produce the accumulation of protein and lipid-rich interstitial fluid and lymphedema [21].

Cholestasis has a pathological effect on the intestinal mucosa [21] [22]. Indeed, short-term cholestatic rats by bile duct ligation suffer a loss of tight junction proteins in the ileal mucosa, with subsequent increased permeability [23] [24]. These significant morphological intestinal mucosa impairments could induce an increased vulnerability of the lacteal lymphatic vessels, because they are located into the intestinal villi, and therefore close to the intestinal mucosa. It has been shown that patients with biliary tract obstruction experience various pathophysiologic changes in the body. Biliary tract obstruction not only induces liver injury but also kidney failure and cardiac dysfunction and hemostatic abnormalities. In addition, biliary tract obstruction also impairs intestinal barrier function and induces intestinal bacterial overgrowth and bacterial translocation [25]. The functions of the intestine are secretory and absorptive, and they play an important role in maintaining homeostasis in the organism. Moreover, intes- 
tinal absorption provides nutrients to the body. It has been found that bile deficiency induced by biliary tract obstruction impairs the intestinal mucosal $\mathrm{HCO}_{3}^{-}$ and $\mathrm{Cl}^{-}$secretion ability, as well as decreases vitamin E, calcium, magnesium, fatty acid, and some drugs intestinal absorption [25].

The lymphatic system of the small intestinal villi originates as a large central vessel or lacteal located at the apical portion of the villi. The lymphatic capillaries of the small intestine are fenestrated. In addition, they are surrounded by a fragmented basement membrane with anchoring filaments attached at the abluminal place [24]. Intestinal lymphatic pathophysiology in cholestatic rats could be related to inflammation, with increased Th1 and Th2 lymphocyte activation in the small bowel and in the mesenteric lymph complex of microsurgical extrahepatic cholestatic rats [26].

The drainage function of lymphatic vessels is crucial for inflammation in the intestinal interstitium. Biologically active factors produced by the intestine during portal hypertension contribute as well as the translocated bacteria to distant organ failure [27] [28]. There is evidence that supports the key role of the intestine and mesenteric lymph nodes in the pathogenesis of multiple organ dysfunction syndrome (MODS) [27] [28]. The inflammation of intestinal mucosa related to portal hypertension, also aggravated by the degree of coexisting hepatic impairment, would cause lymphangiopathy. This alteration of the intestinal lymphatic system is characterized by a deficiency clearance to avoid excess intestinal fluid accumulation and inflammatory mediators. Consequently, inflammation and edema develop, and lymph vessels dilate.

The outstanding role of lymphatic vessels in the etiopathogeny of inflammation has been explained in several excellent reviews [29] [30] [31]. The growth of lymphatic vessels or lymphangiogenesis is actively involved in multiple pathological conditions, including tissue and organ inflammation [32]. In particular, lymphangiogenesis could be advantageous in decreasing inflammation related to lymphedema [24].

The stereological study shown in this paper suggests that the alterations found in the mucosal and submucosal lymphatic microcirculation of cholestatic rats could be attributed to an activation of lymphangiogenesis in response to the intestinal inflammation, mainly in the mucosa. However, further studies would be needed to prove this hypothesis. It has been demonstrated that two members of the VEGF family, VEGF-C and VEGF-D, produce lymphangiogenesis by activation of VEGFR-3, which is expressed by lymphatic endothelial cells in normal adult tissues [33] [34].

The increased volume fraction and length density of lymphatic vessels in the duodenum and ileum, with respect to the jejunum, could correspond to the predominance of the inflammatory response that occurs in portal hypertension in both ends of the small bowel. This characteristic location of the intestinal lymphangiogenesis would be similar to the special intestinal location of the angiogenic response that is developed in portal hypertension. Thus, the excessive angiogenesis in the proximal and distal ends of the intra-abdominal intestinal 
tract, i.e. esophagus and rectum, respectively, induces the development of porto-systemic collateral circulation. This is the reason why it seems appropriate to consider that the excessive lymphangiogenesis in the proximal and distal ends of the small bowel could also represent the development of a mesenteric-systemic lymphatic collateral circulation (Figure 4). The existence of a porto-systemic venous collateral circulation allows noxious substances of intestinal origin, like damage-associated molecular patterns (DAMPs) and pathogen-associated molecular patterns (PAMPs), to reach the systemic circulation [28] [29]. Since the presence of these noxious substances in the systemic circulation severely aggravates the prognosis of the patients with chronic liver disease, the existence of a mesenteric-systemic collateral circulation would therefore represent a new pathophysiological factor in these patients (Figure 5).

In portal hypertension, the excessive angiogenic response and the lymphangiogenic response could be related, among other factors, with the increased mast cells infiltration in the small bowel and mesenteric lymphatic nodes [35] [36]. The human mast cells are a major source of both angiogenic and lymphangiogenic factors. Moreover VEGF-C and VEGD-D produced at sites of inflammation and tumors can amplify mast cells infiltration by interacting with VEGFRs. In addition, mast cell-derived proteases, such as tryptase, MMP2 and MMP9 chymases, can modulate angiogenesis and lymphangiogenesis [35] [37]. Cells of the immune system and their mediators need consideration for future therapeutic manipulation of angiogenesis/lymphangiogenesis in chronic inflammatory disorders and tumors [34].

Portal hypertension related to biliary fibrosis in rats causes extensive mast cell infiltration of the mesenteric lymph complex (MLC) [36]. This massive recruitment

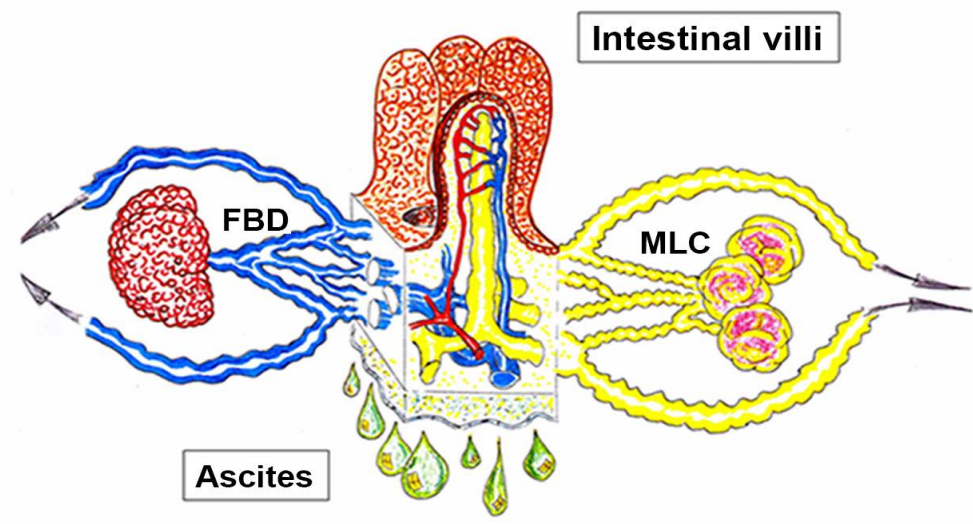

Figure 4. Schematic representation of the collateral splanchno-systemic circulations, venous (left) and lymphatic (right), in rats with microsurgical cholestasis. The acute-on-chronic inflammatory liver disease, secondary to decompensation, in rats with obstructive cholestasis induce portal hypertensive enteropathy, with excessive angiogenic and lymphangiogenic responses, associated with an increased permeability of lymphatic vascular endothelium, interstitial edema, increased permeability of the peritoneal mesothelium and finally, ascites development. FBD: fibrotic biliary disease; MLC: inflamed mesenteric lymph node complex. 


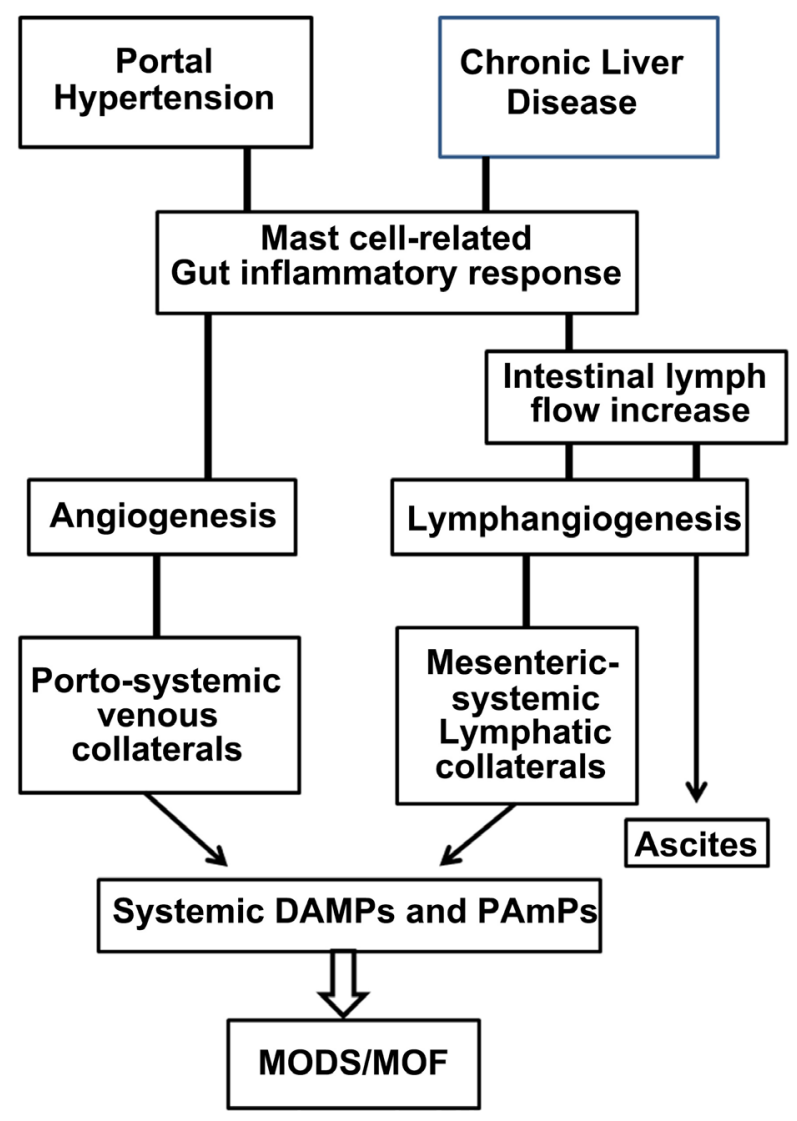

Figure 5. Schematic representation of the etiopathogenic interrelationship between angiogenesis and lymphangiogenesis in the development of splanchno-systemic collaterals in portal hypertension associated to chronic liver disease. DAMPs: damage-associated molecular patterns; MODS: multiorgan dysfunction syndrome; MOF: multiorgan failure; PAMPs: pathogen-associated molecular patterns.

of mast cells in the MLC could in turn impair the lymphatic flow of intestinal origin. Therefore, the mast cell-related mesenteric adenitis in portal hypertension could increase the intestinal lymphatic hypertension. If so, this factor would also induce intestinal inflammation by mechanotransduction and, subsequently, lymphangiogenesis. In particular, chemokines, fundamental mediators of the inflammatory response, would be involved in the lymphatic remodeling process, either vascular or in the lymph nodes [38]. In this way, the hypothesis that the mesenteric-lymphatic collateral circulation is developed through the incidence of two consecutive hits in portal hypertensive rats could be proposed. The first hit would be related to the inflammation produced by portal hypertension, with edema and lymphatic flow increase, while the second hit would be the intestinal lymphatic hypertension, which in turn favor lymphangiogenesis and mesenteric adenitis [38]. Hence, either intestinal angiogenesis or lymphangiogenesis in portal hypertension would have a complementary etiopathogenic role [39].

Lastly, this intestinal lymphatic pathology associated with portal hypertension 
could have an etiopathogenic role in ascites production. Thus, the coupling between portal venous hypertension and mesenteric lymphatic hypertension would induce an intestinal inflammatory response, with being a harmful effect. Maybe this intestinal lymph hyperproduction overwhelms the drainage ability of the neoformed lymphatic collateral circulation in the cholestatic rats. If so, the overloading of lymphatic volume in the interstitium-lymphatic-peritoneum axis would finally cause ascites [6] [39] [40].

\section{Conclusion}

In conclusion, this study demonstrated the development of a lymphatic vascular enteropathy in rats with chronic hepatic disease related to cholestasis. The excessive lymphangiogenesis in the duodenum and ileum could represent the predominance of the inflammatory response in both ends of the small bowel. Then, the consequence would be the development of a mesenteric-systemic lymphatic collateral circulation in this experimental model. Since the porto-systemic venous collateral circulation in the chronic liver insufficiency is developed in the ends of the gastrointestinal tract, the excessive duodeno-ileal lymphangiogenesis could suggest the development of a mesenteric-systemic lymphatic bypass in the chronic portal hypertension. The characterization of the type of intestinal inflammatory response that produces an excessive lymphangiogenesis would lead to a better understanding of the pathophysiological mechanisms involved in portal hypertensive enteropathy. In addition, this understanding would allow for establishing more effective treatments for its severe complications, including collateral lymphatic circulation and ascites.

\section{Funding}

This research was supported by grants from the Mutua Madrileña Foundation (FMM-Ref. No. PA 6977/2009 and AP 105502012).

\section{Acknowledgements}

We would like to acknowledge Carmen Sánchez, from the Autonoma University of Madrid School of Medicine, Department of Anatomy, Histology and Neuroscience, for her valuable technical assistance in preparing the pathological methods in this study and Elizabeth Mascola for translating the manuscript into English.

\section{Conflicts of Interest}

All authors declared no conflict of interest.

\section{References}

[1] Misra, V., Misra, S.P., Dwivedi, M. and Gupta, S.C. (1997) Histomorphometric Study of Portal Hypertensive Enteropathy. American Journal of Clinical Pathology, 108, 652-657. https://doi.org/10.1093/ajcp/108.6.652 
[2] Rondonotti, E., Villa, F., Signorelli, C. and de Francis, R. (2006) Portal Hypertensive Enteropathy. Gastrointestinal Endoscopy Clinics of North America, 16, 277-286. https://doi.org/10.1016/j.giec.2006.01.019

[3] Viggiano, T.R. and Gostout, C.J. (1992) Portal Hypertensive Intestinal Vasculopathy: A Review of the Clinical, Endoscopic and Histopathologic Features. The American Journal of Gastroenterology, 87, 944-954.

[4] Witte, C.L., Witte, M.H. and Dumont, A.E. (1980) Lymph Imbalance in the Genesis and Perpetuation of the Ascites Syndrome in Hepatic Cirrhosis. Gastroenterology, 78, 1059-1068. https://doi.org/10.1016/0016-5085(80)90793-3

[5] Chung, C. and Iwakiri, Y. (2013) The Lymphatic Vascular System in Liver Diseases: Its Role in Ascites Formation. Clinical and Molecular Hepatology, 19, 99-104. https://doi.org/10.3350/cmh.2013.19.2.99

[6] Aller, M.A., Prieto, I., Argudo, S., de Vicente, F., Santamaría, L., de Miguel, M.P., Arias, J.L. and Arias, J. (2010) The Interstitial Lymphatic Peritoneal Mesothelium Axis in Portal Hypertensive Ascites: When in Danger, Go Back to the Sea. International Journal of Inflammation, 2010, Article ID: 148689.

https://doi.org/10.4061/2010/148689

[7] Aller, M.A., Arias, J.L., Cruz, A. and Arias, J. (2007) Inflammation: A Way to Understanding the Evolution of Portal Hypertension. Theoretical Biology and Medical Modelling, 4, 44. https://doi.org/10.1186/1742-4682-4-44

[8] Mehta, G., Gusto, T., Mookerjee, R.P., Garcia-Pagan, J.C., Fallon, M.B., Shah, V.H., Moreau, R. and Jalan, R. (2014) Inflammation and Portal Hypertension-The Undiscovered Country. Journal of Hepatology, 61, 155-163.

https://doi.org/10.1016/j.jhep.2014.03.014

[9] Aller, M.A., Lorente, L., Alonso, S. and Arias, J. (1993) A Model of Cholestasis in the Rat, Using a Microsurgical Technique. Scandinavian Journal of Gastroenterology, 28, 10-14. https://doi.org/10.3109/00365529309096038

[10] Aller, M.A., Prieto, I., Cruz, A., Losada, M., Arias, J.I., Garcia-Dominguez, J., Argudo, S., Arias, J.L. and Arias, J. (2009) Extrahepatic Cholestasis. In: Aller, M.A. and Arias, J., Eds., Microsurgery in Liver Research, Bentham Scientific, Sharjah, e-Books, 137-156.

[11] Baluk, P. and McDonald, D.M. (2008) Marker for Microscopic Imaging of Lymphangiogenesis and Angiogenesis. Annals of the New York Academy of Sciences, 1131, 1-12. https://doi.org/10.1196/annals.1413.001

[12] Pepper, M.S. and Skobe, M. (2013) Lymphatic Endothelium: Morphological, Molecular and Functional Properties. The Journal of Cell Biology, 163, 209-213. https://doi.org/10.1083/jcb.200308082

[13] Hong, Y.K. and Detmar, M. (2003) Prox 1, Master Regulator of the Lymphatic Vasculature Phenotype. Cell and Tissue Research, 314, 85-92. https://doi.org/10.1007/s00441-003-0747-8

[14] Schulte-Merker, S., Sabine, A. and Petrova, T.V. (2011) Lymphatic Vascular Morphogenesis in Development, Physiology, and Disease. The Journal of Cell Biology, 193, 607-618. https://doi.org/10.1083/jcb.201012094

[15] Alitalo, K. (2011) The Lymphatic Vasculature in Disease. Nature Medicine, 17, 1371-1380. https://doi.org/10.1038/nm.2545

[16] Ohno, N., Terada, N., Bai, Y., Saitoh, S., Nakazawa, T., Nakamura, N., Naito, I., Fujii, Y., Katoh, R. and Ohno, S. (2008) Application of Cryobiopsy to Morphological and Immunohistochemical Analyses of Xenografted Human Lung Cancer Tissues and Functional Blood Vessels. Cancer, 113, 1068-1079. 
https://doi.org/10.1002/cncr.23701

[17] Ohno, N., Terada, N. and Ohno, S. (2006) Histochemical Analyses of Living Mouse Liver under Different Hemodynamic Conditions by "In Vivo Cryotechnique". Histochemistry and Cell Biology, 126, 389-398.

https://doi.org/10.1007/s00418-006-0173-6

[18] Santamaria, L., Ingelmo, I., Ruiz, J., Teba, F., Herranz, L.M., Montalban, G., Martin, R., Codesal, J., Pozuelo, J.M., Rodriguez, R. and Riansares Arriazu, R. (2009) Stereological Estimate of the Length of Microvessels and the Number, Proliferation and Apoptosis of Endothelial Cells in Prostate Cancer. The Open Prostate Cancer Journal, 2, 46-53. https://doi.org/10.2174/1876822900902010046

[19] Sterio, D.C. (1984) The Unbiased Estimation of Number and Sizes of Arbitrary Particles Using the Dissector. Journal of Microscopy, 134, 127-136. https://doi.org/10.1111/j.1365-2818.1984.tb02501.x

[20] Lokmic, Z. and Mitchell, G.M. (2011) Visualization and Stereological Assessment of Blood and Lymphatic Vessels. Histology and Histopathology, 26, 781-796.

[21] Gouma, D.J., Coelho, J.C., Fisher, F.D., Komura, M., Yamaguchi, K., Kuroki, S. and Tanaka, M. (1986) Endotoxemia after Relief of Biliary Obstruction by Internal and External Drainage in Rats. The American Journal of Surgery, 151, 476-479. https://doi.org/10.1016/0002-9610(86)90107-8

[22] Quraishy, M.S., Chescoe, D., Mullervy, J., Coates, M., Hinton, R.H. and Bailey, M.E. (1996) Influence of the Gut Microflora and of Biliary Constituents on Morphological Changes in the Small Intestine in Obstructive Jaundice. HPB Surgery, 10, 11-20. https://doi.org/10.1155/1996/43159

[23] Porticansa, P., Grattaguano, I., Testini, M., Caruso, M.L., Wang, D.Q., Moschetta, A., Calamita, G., Vacca, M., Valentini, A.M., Renna, G., Lissidini, G. and Palasciano, G. (2007) Parallel Intestinal and Liver Injury during Early Cholestasis in the Rat: Modulation by Bile Salts and Antioxidants. Free Radical Biology \& Medicine, 42, 1381-1391. https://doi.org/10.1016/j.freeradbiomed.2007.01.039

[24] Abouelkheir, G.R., Upchurch, B.D. and Rutkowski, J.M. (2017) Lymphangiogenesis: Fuel, Smoke, or Extinguisher of Inflammation's Fire? Experimental Biology and Medicine, 242, 884-895. https://doi.org/10.1177/1535370217697385

[25] Du, Y., Chen, H., Xuan, Z., Song, W., Hong, L., Guo, D., Li, H., Tuo, B., Zheng, S. and Song, P. (2016) Bile Deficiency Induces Changes in Intestinal Glucose Absorption in Mice. Surgery, 160, 1496-1507. https://doi.org/10.1016/j.surg.2016.05.040

[26] Garcia-Dominguez, J., Aller, M.A., Garcia, C., de Vicente, F., Corcuera, M.T., Gómez-Aguado, F., Alonso, M..J., Vara, E. and Arias, J. (2010) Splanchnic Th(2) and $\mathrm{Th}(1)$ Cytokine Redistribution in Microsurgical Cholestatic Rats. Journal of Surgical Research, 162, 203-212. https://doi.org/10.1016/j.jss.2009.06.010

[27] Deitch, E.A. (2001) Role of the Gut Lymphatic System in Multiple Organ Failure. Current Opinion in Critical Care, 7, 92-98. https://doi.org/10.1097/00075198-200104000-00007

[28] Deitch, E.A. (2002) Bacterial Translocation or Lymphatic Drainage of Toxic Products from the Gut: What Is Important in Human Beings? Surgery, 131, 241-244. https://doi.org/10.1067/msy.2002.116408

[29] Cueni, L.N. and Detmar, M. (2008) The Lymphatic System in Health and Disease. Lymphatic Research and Biology, 6, 109-122. https://doi.org/10.1089/lrb.2008.1008

[30] Tammela, T. and Alitalo, K. (2010) Lymphangiogenesis: Molecular Mechanisms and Future Promise. Cell, 140, 460-476. https://doi.org/10.1016/j.cell.2010.01.045 
[31] Kim, H., Kataru, R.P. and Koh, G.Y. (2012) Regulation and Implications of Inflammatory Lymphangiogenesis. Trends in Immunology, 33, 353-356. https://doi.org/10.1016/j.it.2012.03.006

[32] Lee, J., Gray, A., Yuan, J., Luoh, S.M., Avraham, H. and Wood, W.I. (1996) Vascular Endothelial Growth Factor-Related Protein: A Ligand and Specific Activator of the Tirosine Kinase Receptor Flr4. Proceedings of the National Academy of Sciences of the United States of America, 93, 1988-1992. https://doi.org/10.1073/pnas.93.5.1988

[33] Martinez-Corral, I., Olmeda, D., Dieguez-Hurtad, R., Tammela, T., Alitalo, K. and Ortega, S. (2012) In Vivo Imaging of Lympatic Vessels in Development, Wound Healing, Inflammation, and Tumor Metastasis. Proceedings of the National Academy of Sciences of the United States of America, 109, 6223-6228. https://doi.org/10.1073/pnas.1115542109

[34] Prieto, I., Aller, M.A., Santamaría, L., Nava, M.P., Madero, R., Pérez-Robledo, J.P. and Arias, J. (2005) Prehepatic Portal Hypertension Produces Increased Mast Cell Density in the Small Bowel and in Mesenteric Lymph Nodes in the Rat. Journal of Gastroenterology and Hepatology, 20, 1025-1031. https://doi.org/10.1111/j.1440-1746.2005.03831.x

[35] Moquillaza, L.M., Aller, M.A., Nava, M.P., Santamaría, L., Vergara, P. and Arias, J. (2010) Partial Hepatectomy, Partial Portal Vein Stenosis and Mesenteric Lymphadenectomy Increase Splanchnic Mast Cell Infiltration in the Rat. Acta Histochemica, 112, 372-382. https://doi.org/10.1016/j.acthis.2009.03.002

[36] Aller, M.A., Arias, J.L., García-Domínguez, J., Arias, J.I., Durán, M. and Arias, J. (2008) Experimental Obstructive Cholestasis: The Wound-Like Inflammatory Liver Response. Fibrogenesis Tissue Repair, 1, 6. https://doi.org/10.1186/1755-1536-1-6

[37] Isaza-Restrepo, A., Martin-Saavedra, J.S., Velez-Leal, J.L., Vargas-Barato, F. and Riveros-Dueñas, R. (2018) Peritoneum: Beyond the Tissue-A Review. Frontiers in Physiology, 9, 738. https://doi.org/10.3389/fphys.2018.00738

[38] Farnsworth, R.H., Karnezis, T., Maciburko, S.J., Mueller, S.N. and Stacker, S.A. (2019) The Interplay Between Lymphatic Vessels and Chemokines. Frontiers in Immunology, 10, 518. https://doi.org/10.3389/fimmu.2019.00518

[39] Sanchez-Patan, F., Anchuelo, R., Corcuera, M.T., Casado, I., Gómez-Aguado, F., Aller, M.A., Cruz, A., Alonso, M.J. and Arias, J. (2008) Biliary Fibrosis in Microsurgical Extrahepatic Cholestasis in the Rat. Microsurgery, 28, 361-366.

https://doi.org/10.1002/micr.20495

[40] Varricchi, G., Loffredo, S., Galdiero, M.R., Marone, G., Cristinziano, L., Granata, F. and Marone, G. (2018) Innate Effector Cells in Angiogenesis and Lymphangiogenesis. Current Opinion in Immunology, 53, 1521-1560.

https://doi.org/10.1016/j.coi.2018.05.002 